\title{
Data fusion technique using wavelet transform and taguchi methods for automatic landslide detection from airborne laser scanning data and quickbird satellite imagery
}

\begin{abstract}
Landslide mapping is indispensable for efficient land use management and planning. Landslide inventory maps must be produced for various purposes, such as to record the landslide magnitude in an area and to examine the distribution, types, and forms of slope failures. The use of this information enables the study of landslide susceptibility, hazard, and risk, as well as of the evolution of landscapes affected by landslides. In tropical countries, precipitation during the monsoon season triggers hundreds of landslides in mountainous regions. The preparation of a landslide inventory in such regions is a challenging task because of rapid vegetation growth. Thus, enhancing the proficiency of landslide mapping using remote sensing skills is a vital task. Various techniques have been examined by researchers. This study uses a robust data fusion technique that integrates high-resolution airborne laser scanning data (LiDAR) with high-resolution QuickBird satellite imagery (2.6$\mathrm{m}$ spatial resolution) to identify landslide locations in Bukit Antarabangsa, Ulu Klang, Malaysia. This idea is applied for the first time to identify landslide locations in an urban environment in tropical areas. A wavelet transform technique was employed to achieve data fusion between LiDAR and QuickBird imagery. An object-oriented classification method was used to differentiate the landslide locations from other land use/covers. The Taguchi technique was employed to optimize the segmentation parameters, whereas the rule-based technique was used for object-based classification. In addition, to assess the impact of fusion in classification and landslide analysis, the rule-based classification method was also applied on original QuickBird data which have not been fused. Landslide locations were detected, and the confusion matrix was used to examine the proficiency and reliability of the results. The achieved overall accuracy and kappa coefficient were $90.06 \%$ and 0.84 , respectively, for fused data. Moreover, the acquired producer and user accuracies for landslide class were $95.86 \%$ and $95.32 \%$, respectively. Results of the accuracy assessment for QuickBird data before fusion showed $65.65 \%$ and 0.59 for overall accuracy and kappa coefficient, respectively. It revealed that fusion made a significant improvement in classification results. The direction of mass movement was recognized by overlaying the final landslide classification map with LiDAR-derived slope and aspect factors. Results from the tested site in a hilly area showed that the proposed method is easy to implement, accurate, and appropriate for landslide mapping in a tropical country, such as Malaysia.
\end{abstract}

Keyword: Fusion; Landslide; Laser scanning data (LiDAR); Malaysia; Remote sensing; Rule-based; Taguchi; Wavelet transform 